\title{
EPR Studies of Carbonized Cellulose Oxygen Interactions
}

\author{
B. Pilawa ${ }^{a, *}$, R. Pietrzak ${ }^{b}$, H. Wachowska ${ }^{b}$ And K. Babę ${ }^{c}$ \\ ${ }^{a}$ Institute of Coal Chemistry, Polish Academy of Sciences \\ Sowińskiego 5, 44-121 Gliwice, Poland \\ ${ }^{b}$ Laboratory of Coal Chemistry and Technology, Faculty of Chemistry \\ Adam Mickiewicz University, Grunwaldzka 6, 60-780 Poznań, Poland \\ ${ }^{c}$ Institute of Chemical Wood Technology, Agricultural Academy of Poznań \\ Wojska Polskiego 38/42, 60-637 Poznań, Poland
}

EPR examination of cellulose carbonized at $400^{\circ} \mathrm{C}$ and $600^{\circ} \mathrm{C}$ has been performed. The aim of this work was to search a sample useful as the oximetric probe in biology and medicine. The higher paramagnetic centers concentration characterizes cellulose carbonized at $600^{\circ} \mathrm{C}$, which was found as the sensitive oximetric probe. Quasi-chemical bonds between cellulose molecules and paramagnetic $\mathrm{O}_{2}$ molecules decrease paramagnetic centers concentration. A linear increase in paramagnetic centers concentration and a linear decrease in EPR linewidth with increasing time of sample evacuation were observed for cellulose carbonized at $600^{\circ} \mathrm{C}$. Oxygen affects spin-lattice interactions in carbonized cellulose.

PACS numbers: $61.66 . \mathrm{Hq}, 76.30 . \mathrm{Rn}$

\section{Introduction}

EPR spectra of organic systems strongly depend on existence of paramagnetic oxygen molecules in the environment of the samples [1]. Oxygen $\mathrm{O}_{2}$ affects shape, linewidths, and amplitudes of resonance curves. Spin-lattice relaxation processes change in samples in air. Both broadening and narrowing of broad $\left(\Delta B_{\mathrm{pp}}\right.$ : 0.50-0.90 $\mathrm{mT}$ ) EPR lines of macerals carbonized at $300-650^{\circ} \mathrm{C}$ were observed in air [2]. Narrowing of the narrow EPR lines $\left(\Delta B_{\mathrm{pp}}: 0.06-0.34 \mathrm{mT}\right)$ of thermally

*corresponding author; e-mail: pilawa@karboch.gliwice.pl 
decomposed macerals dominates in air [2]. A decrease in concentration of paramagnetic centers with narrow $\left(\Delta B_{\mathrm{pp}}: 0.29-0.34 \mathrm{mT}\right)$ EPR lines caused by maceral interactions with oxygen is known $[1,2]$. A decrease in spin-lattice relaxation time for macerals in air is also measured [2].

In this work interactions of carbonized cellulose with paramagnetic oxygen are examined. The aim of this study was to find oxygen sensitive probe for biological systems. It is expected that thermally treated cellulose is inert for cell cultures, whereas its EPR lines is susceptible for oxygen effect.

\section{Experimental}

EPR spectra of the original cellulose, and cellulose carbonized at $400^{\circ} \mathrm{C}$ and $600^{\circ} \mathrm{C}$ for samples evacuated 24 hours were measured. Cellulose carbonized at $600^{\circ} \mathrm{C}$ and evacuated $1-5$ hours were studied by EPR method.

EPR measurements were performed using an X-band (9.3 GHz) spectrometer with modulation of magnetic field $100 \mathrm{kHz}$. The microwave frequency was measured. The $g$-factors, linewidths $\Delta B_{\mathrm{pp}}$, amplitudes, concentrations of paramagnetic centers were obtained.

Ultramarine was used as the reference of paramagnetic centers concentration. Area under absorption curve was obtained as double integration of the first derivative EPR line. The influence of microwave power on EPR lines was analyzed.

\section{Results and discussion}

To find a cellulose sample useful as the oximetric probe, the paramagnetic centers in the original cellulose and cellulose carbonized at $400^{\circ} \mathrm{C}$ and $600^{\circ} \mathrm{C}$ were tested. The original cellulose sample in vacuum reveals EPR lines with a linewidth of $0.30 \mathrm{mT}$ and low paramagnetic centers concentration $\left(1.0 \times 10^{16} \mathrm{spin} / \mathrm{g}\right)$. Concentrations of paramagnetic centers in cellulose carbonized at $400^{\circ} \mathrm{C}$ and $600^{\circ} \mathrm{C}$ for the evacuated samples were determined as $4.7 \times 10^{19} \mathrm{spin} / \mathrm{g}$ and $17.7 \times 10^{19} \mathrm{spin} / \mathrm{g}$, respectively. EPR linewidth $\Delta B_{\mathrm{pp}}$ of cellulose decreases from $0.56 \mathrm{mT}$ to $0.43 \mathrm{mT}$ with increasing heating temperature from $400^{\circ} \mathrm{C}$ to $600^{\circ} \mathrm{C}$. The $g$-factors in the range of 2.0031-2.0035 were calculated from the resonance condition. EPR lines of cellulose carbonized at $600^{\circ} \mathrm{C}$ saturate at higher microwave power than those of cellulose carbonized at $400^{\circ} \mathrm{C}$. Faster spin-lattice relaxation processes exist in the sample carbonized at $600^{\circ} \mathrm{C}$.

Cellulose carbonized at $600^{\circ} \mathrm{C}$ was chosen as oximetric probe. Because of higher paramagnetic centers concentration and formation of multi-ring aromatic structures during thermal decomposition, probability of interactions with $\mathrm{O}_{2}$ molecules is higher for cellulose carbonized at $600^{\circ} \mathrm{C}$. The linear decrease in paramagnetic centers concentration with increasing oxygen concentration in environment of cellulose carbonized at $600^{\circ} \mathrm{C}$ was observed (Fig. 1). Quasi-chemical 


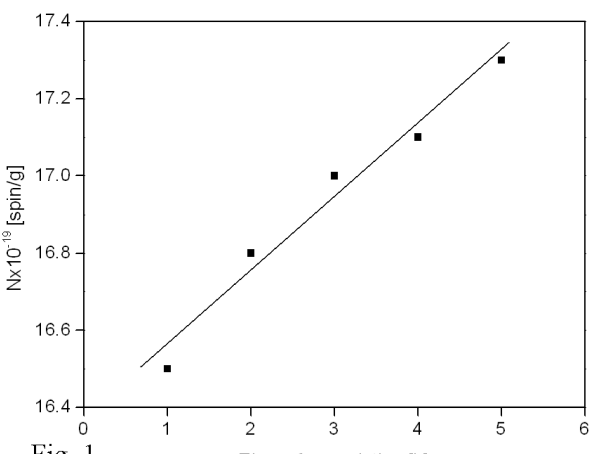

Fig. 1

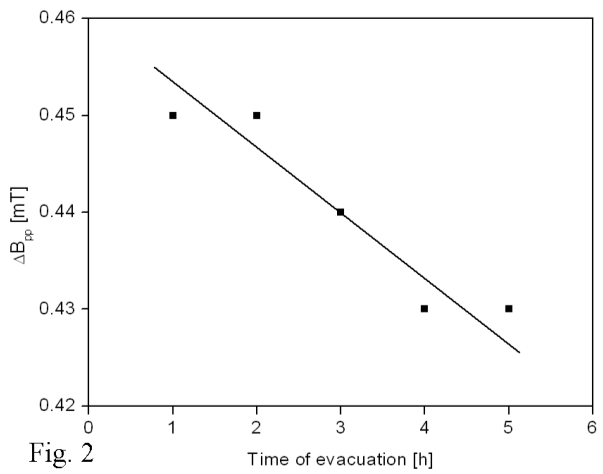

Fig. 2

Fig. 1. Changes of paramagnetic centers concentration in cellulose carbonized at $600^{\circ} \mathrm{C}$ with increasing time of sample evacuation.

Fig. 2. Changes of linewidth $\Delta B_{\mathrm{pp}}$ of EPR line of cellulose carbonized at $600^{\circ} \mathrm{C}$ with increasing time of sample evacuation.
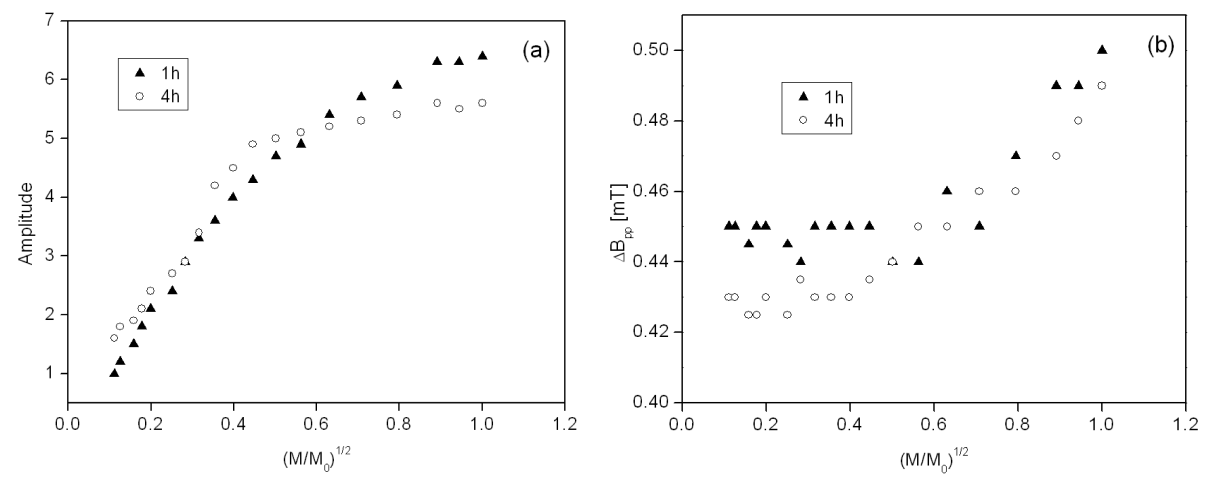

Fig. 3. Influence of microwave power on amplitudes (a) and linewidths $\Delta B_{\mathrm{pp}}$ (b) of EPR lines of cellulose carbonized at $600^{\circ} \mathrm{C}$ for sample evacuated 1 and 4 hours.

bonds between the sample and $\mathrm{O}_{2}$ are formed. The broadening of EPR lines of cellulose sample for higher oxygen concentrations was observed (Fig. 2).

Changes of amplitudes and linewidths of EPR lines in carbonized cellulose with microwave power for samples evacuated 1 and 4 hours are presented in Fig. 3a, b, respectively. EPR lines of cellulose carbonized at $600^{\circ} \mathrm{C}$ are homogeneously broadened (Fig. 3). EPR lines of cellulose sample with lower oxygen content in the environment (longer evacuated) saturate earlier than EPR lines of the sample with a higher oxygen content in the environment (Fig. 3a).

\section{Conclusions}

Spin-lattice and spin-spin interactions, and paramagnetic centers concentration in cellulose carbonized at $600^{\circ} \mathrm{C}$ depend on the oxygen concentration in 
the environment of the sample. Paramagnetic oxygen molecules cause fastening of spin-lattice relaxation processes in the carbonized cellulose. The linear increase in linewidth and decrease in paramagnetic centers concentration with increasing time of sample evacuation were observed. The electron paramagnetic resonance results pointed out that carbonized cellulose is useful as probe in oximetry.

\section{References}

[1] B. Pilawa, A.B. Więckowski, Fuel 76, 1173 (1997).

[2] B. Pilawa, Evolution of Paramagnetic Centers during Thermal Decomposition of Macerals, WSP, Zielona Góra 2000 (in Polish). 